UDC 539

\title{
$R \& D$ status of pulse shape analysis for short-lived decay of superheavy elements in GARIS-II
}

\author{
Yamaki S. ${ }^{{ }^{*}}$, Morimoto K. ${ }^{1}$, Kaji D. ${ }^{1}$, Wakabayashi Ya. ${ }^{1}$, Takeyama M. ${ }^{3}$, \\ Tanaka K. ${ }^{1,4}$, Tanaka T. ${ }^{1}$, Baba H. ${ }^{1}$, Yamaguchi T. ${ }^{2}$, Suzuki T. ${ }^{2}$, Morita K. ${ }^{1}$

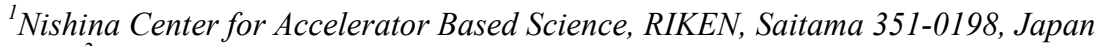 \\ ${ }^{2}$ Department of Physics, Saitama University, Saitama 338-8570, Japan \\ ${ }^{3}$ Graduate School of Science and Engineering, Yamagata University, Yamagata, 990-8560, Japan \\ ${ }^{4}$ Faculty of Science and Technology, Tokyo University of Science, Chiba 278-8510, Japan \\ ${ }^{5}$ Department of Physics, Kyusyu University, Fukuoka 812-8581, Japan \\ e-mail: sayaka.yamaki@riken.jp
}

\begin{abstract}
To search undiscovered superheavy elements, a series of commissioning experiments for the new gas-filled separator GARIS-II at RIKEN was recently carried out. A new data acquisition method was tested to study the short-lived $\alpha$ decay. Both the $\alpha$ decay and evaporation residues were detected by the silicon detectors, and the preamplified waveforms were then directly registered by a flash ADC and analyzed by means of a pulse shape analysis. This paper describes the identification of two sequential pulses (pileup events) from short-lived $\alpha$ decays and the determination of their energy and time difference by the template fitting method. The performance of the present system was evaluated by using a simulated data set and was successfully employed for online experimental data such as ${ }^{220} \mathrm{Ac} \rightarrow{ }^{216} \mathrm{Fr}$.
\end{abstract}

Key words: Superheavy elements, Pulse-shape analysis, $\alpha$ decays, short half-life

PACS numbers: 25.70.Ji, 23.60.+e, 29.85.Fj

\section{Introduction}

A project for synthesizing the 113th element by the cold-fusion reaction ${ }^{209} \mathrm{Bi}\left({ }^{70} \mathrm{Zn}, \mathrm{n}\right){ }^{278} 113$ started at RIKEN in 2003. Three decay chains originating from ${ }^{278} 113$ were observed in 2004, 2005, and 2012 [1-3] . This project came to an end with the observation of the third decay chain. Our focus is now on the search for undiscovered heavier new elements such as $Z=$ 119 and beyond. A series of commissioning experiments for the new gas-filled separator GARIS-II [4] designed for hot fusion reaction has almost been completed.

\section{Data acquisition and analysis systems for the short lived decay}

The half-lives of undiscovered isotopes are calculated by many theoretical approaches. As for ${ }^{298} 120$, typically if the $Q_{\alpha}$ value is around $13 \mathrm{MeV}$, the half-life will be on the order of microseconds [5, 6]. However, the measurement of the decay properties of such short-lived decays is difficult with the existing data acquisition system. The preampilfied waveforms have a long tail; therefore, in the case of a short-lived decay, two sequential pulses pile up. These piled-up pulses are shaped together in the shaping amplifier; thus, the information of an individual decay, such as the decay energy and time, will be lost. We overcome this problem by using a hybrid system utilizing analog and digital data acquisition systems; the former is the original system, whereas the latter is specialized for pileup events, where the time difference between two sequential events $(\Delta T)$ is less than $10 \mu \mathrm{s}$. The waveforms from the preamplifiers of the silicon detectors are directly registered with flash ADCs to avoid the summing phenomenon. The readout system of GARIS-II is explained in [7].

Subsection 2.1 describes a method for analyzing the preamplifier waveforms to extract the decay information, and subsection 2.2 presents a performance evaluation of this system.

\subsection{Pulse shape analysis}

The left panel of Figure 1 shows preamplified piled-up pulses. Case (i) shows that both the first and second $\alpha$ particles are stopped in the double-sided silicon strip detector (DSSD). Case (ii) shows that the first $\alpha$ particle is stopped in the DSSD, and the second $\alpha$ particle escapes from DSSD; therefore, only part of the $\alpha$ energy is deposited in the DSSD, and vise-versa for case (iii). When the evaporation 
residues are implanted and the subsequently decaying $\alpha$ particles are detected, the waveforms are also similar to case (ii). The decay properties are determined through a two-stage pulse shape analysis
(PSA). The first stage is event identification to determine whether the detected pulses result from single events or pileup events, and the second stage is template fitting.

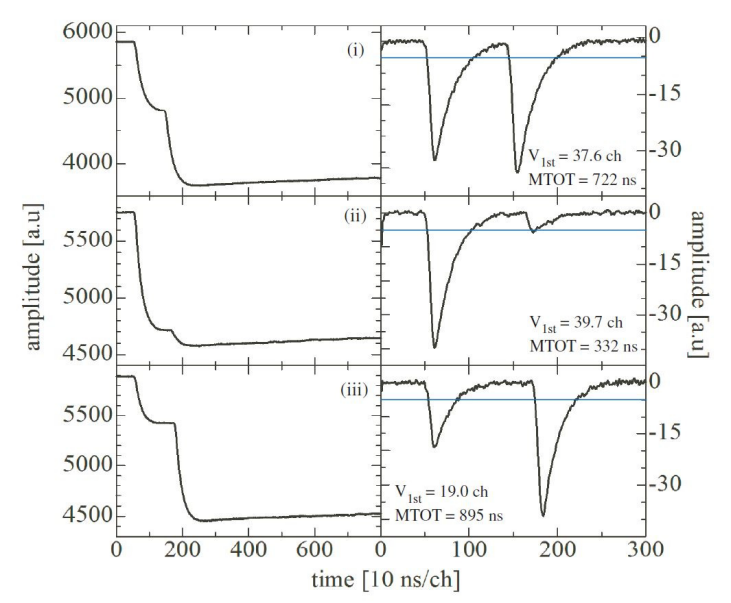

Figure 1 - Left: Original waveforms of pileup events. The sampling period is $8 \mu \mathrm{s}(10 \mathrm{~ns} / \mathrm{ch})$.

Right: the solid lines indicate the derivatives of the waveforms shown on the left.

The blue line shows the threshold $\left(v_{t h}\right)$ for calculating the mean of the time over threshold (MTOT).

The values of the first-pulse amplitude $\left(V_{1 s t}\right)$ and MTOT are shown for each case
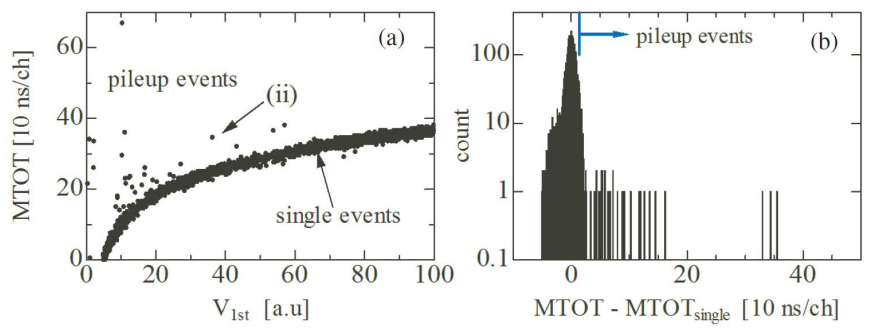

Figure $2-$ (a) Relation between the MTOT and $V_{1 s t}$.

Single events are distributed around the logarithmic line. Pileup events are found above the line.

The data point for case (ii) in Figure 1 is indicated by an arrow. (b) Histogram of the distribution of MTOT $-M T O T_{\text {single }}$.

The threshold for discriminating single and pileup events is set at $14.2 \mathrm{~ns}$

The graphs on the right side of Figure 1 show the derivatives of the waveforms shown in the graphs on the left side. The identification of single or pileup events is performed by calculating the mean of the time over threshold (MTOT) [8] of these waveforms. The MTOT is defined as

$$
\mathrm{MTOT}=\frac{\sum_{t=0}^{t_{m}} w_{t}}{\sum_{t=0}^{t_{m}} w_{t}}-T, w_{t}=\left\{\begin{array}{l}
1\left(v_{t} \leq v_{t h .}\right) \\
0\left(v_{t}>v_{t h .}\right)
\end{array},\right.
$$

where $t$ is the time $(10 \mathrm{~ns} / \mathrm{ch}), t_{m}$ is the sampling period, $v_{t}$ is the amplitude, $v_{t h}$ is the amplitude threshold, and $T$ is the minimum $t$ when $w_{t}=1$. For pileup events, the MTOT depends on the $\Delta T$ and energy ratio $\left(E_{1 s t} / E_{2 n d}\right)$ between two pulses. The MTOT for each case is presented in Figure 1. In general, the MTOT is small when the $\Delta T$ is short or when the pulse height of second pulse is smaller than that of first pulse, as shown in case (ii).

The MTOT is compared with the pulse height of the first pulse $\left(V_{1 s t}\right)$. The values of $V_{1 s t}$ are also shown in Figure 1. Figure 2(a) shows the relation between the MTOT and $V_{1 s t}$. Single pulses are distributed along the logarithmic line

$$
\mathrm{MTOT}_{\text {single }}=a \cdot \log \left(V_{1 s t}-b\right)
$$


where $a$ and $b$ are derived for each stripped channel of the DSSD. Figure 2(b) shows a histogram of the difference of the MTOT from the logarithmic line, i.e., MTOT - MTOT $T_{\text {single }}$. The Gaussian distribution denotes single events, and the tail on the right side denotes pileup events. The threshold for discriminating single and pileup events is set at 14.2 ns ( $\sim 2 \sigma$ of the single-event peak).

Both the single and pileup waveforms are fitted with the template function. The fitting formula is

$$
f(t)=A[0] \cdot \text { template }(t-A[1])+\text { baseline, }
$$

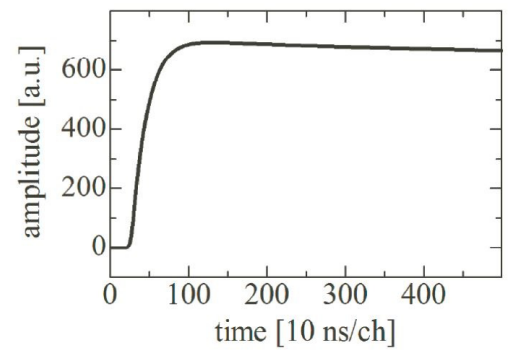

Figure 3 - The left panel shows the template function created by averaging many waveforms.

The right panel shows the reduced $\chi^{2}$ distribution for the fitting of single pulses.

Each pulse of a pileup event is fitted separately. Considering $E_{\text {templ. }}$ as the calibrated energy of the template, the energy of the fitted pulse is deduced by $A[0] \cdot E_{\text {templ. }}$. In addition, the decay time of the second event is determined as $A_{2 h d}$ [1] $-A_{1 s t}$ [1].

\subsection{Performance evaluation}

To evaluate the performance of the PSA, a simulation study was performed. Pileup events were artificially created by summing two single pulses obtained in an online experiment. Pulses with energies of 1-20 MeV were selected. The $\Delta T$ were fixed at 50,100,200, 300, 400, 500, 600, and $700 \mathrm{~ns}$.

Figure 4 shows the efficiency of pileup

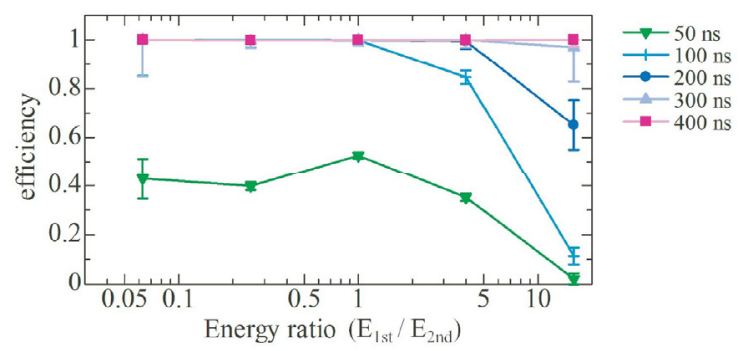

Figure 4 - The efficiency of pileup-event separation by the MTOT method.

This is the efficiency for identifying every event as a pileup event and not as a single event where the free parameters $A[0]$ and $A[1]$ are the pulse height and time offset, respectively. The left panel of Figure 3 shows a typical example of the template function, which is created by averaging many waveforms. The right panel of Figure 3 shows the reduced $\chi^{2}$ distribution for the fitting of single pulses. The number of events for creating a template was varied among the values of 2, 5, 10, and 200, and the energy range was 5-6 MeV. Under the present conditions, only five events are sufficient for the creation of an accurate template function.

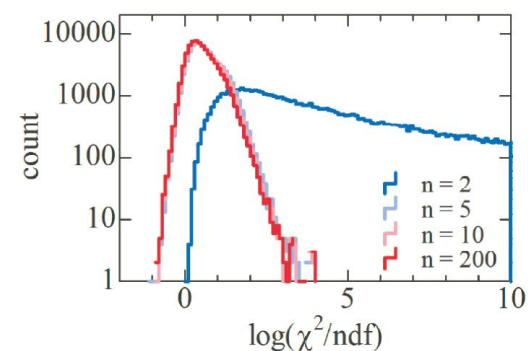

separation by the MTOT method. Here, the efficiency is defined as the ratio of the number of identified pileup events relative to the total number of events. The efficiency is almost $100 \%$ down to a $\Delta T$ of $300 \mathrm{~ns}$ for $E_{1 s t} / E_{2 n d}$ ranging from 0.06 to 16 . Figure 5 shows the energy and time-difference accuracy of the template fitting. Events that have the correct energy $\pm 380 \mathrm{keV}$ and correct $\Delta T \pm 30 \mathrm{~ns}$ were considered to be successfully fitted. The results are again satisfactory unless the $\Delta T$ is small. Note that for both the efficiency and accuracy of the PSA, the performance deteriorates when the $E_{1 s t} / E_{2 n d}$ is large.

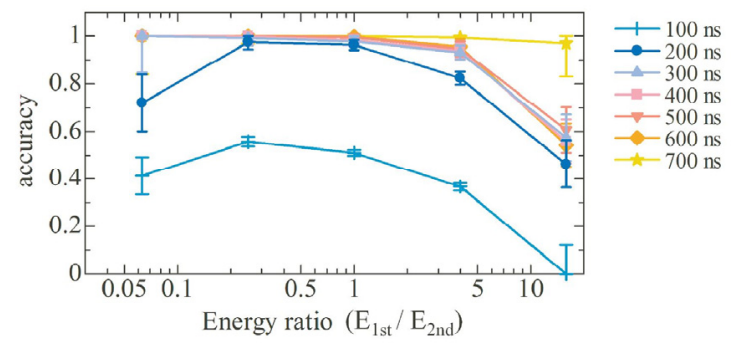

Figure 5 - The accuracy of the template-fitted waveforms. As criteria for successful fitting, an energy width of $\pm 380 \mathrm{keV}$ and a $\Delta T$ width of $\pm 30 \mathrm{~ns}$ with respect to the true energy and $\Delta T$, respectively, were used 
The PSA was applied to online experimental data. Figure 6 shows some examples of ${ }^{220} \mathrm{Ac} \rightarrow{ }^{216} \mathrm{Fr}$ pileup events. The top graphs show the derivative waveforms, the middle graphs show the fittings of the first pulses $\left({ }^{220} \mathrm{Ac}\right)$, and the bottom graphs show the fittings of the second pulses $\left({ }^{216} \mathrm{Fr}\right)$. The values of the extracted $\alpha$ decay energies and decay times are also indicated in the figure. These pileup events were assigned by confirming the decay chain of ${ }^{224} \mathrm{~Pa}$ $\rightarrow{ }^{220} \mathrm{Ac}+{ }^{216} \mathrm{Fr} \rightarrow{ }^{212} \mathrm{At}$ with the analog system. For these four events, the decay energies of the pileup events from ${ }^{220} \mathrm{Ac}$ and ${ }^{216} \mathrm{Fr}$ measured by the analog system are $16.8,16.7,16.7$, and $16.8 \pm 0.1 \mathrm{MeV}$ from the left panel to right panel. The known values of the $\alpha$ decay energies for ${ }^{220} \mathrm{Ac}$ and ${ }^{216} \mathrm{Fr}$ are 7.86, 7.71, and $7.79 \mathrm{MeV}\left(I_{\alpha} \geq 10\right)$ and $9.01 \mathrm{MeV}$, respectively, and the half-life for ${ }^{216} \mathrm{Fr}$ is $0.7 \mu s$ [9]. The cases shown in the three left-most panels are in good agreement with reference values. As the energy resolution depends on the $\Delta T$ of pileup events, the best resolution for $\Delta T<200 \mathrm{~ns}$ was $0.21 \mathrm{MeV}$ (FWHM). In the right-most panel, the waveform could not be properly differentiated because of the slow rise time and noise. This situation also leads to the misidentification of the pulse type, i.e., single or pileup. Even if identification is successful, the energy and decay time are also likely to deviate from the correct values, as in this example. For better efficiency and accuracy, further improvements in the measurement conditions, such as a noise reduction or the tuning of the rise time of the preamplifier, are required.
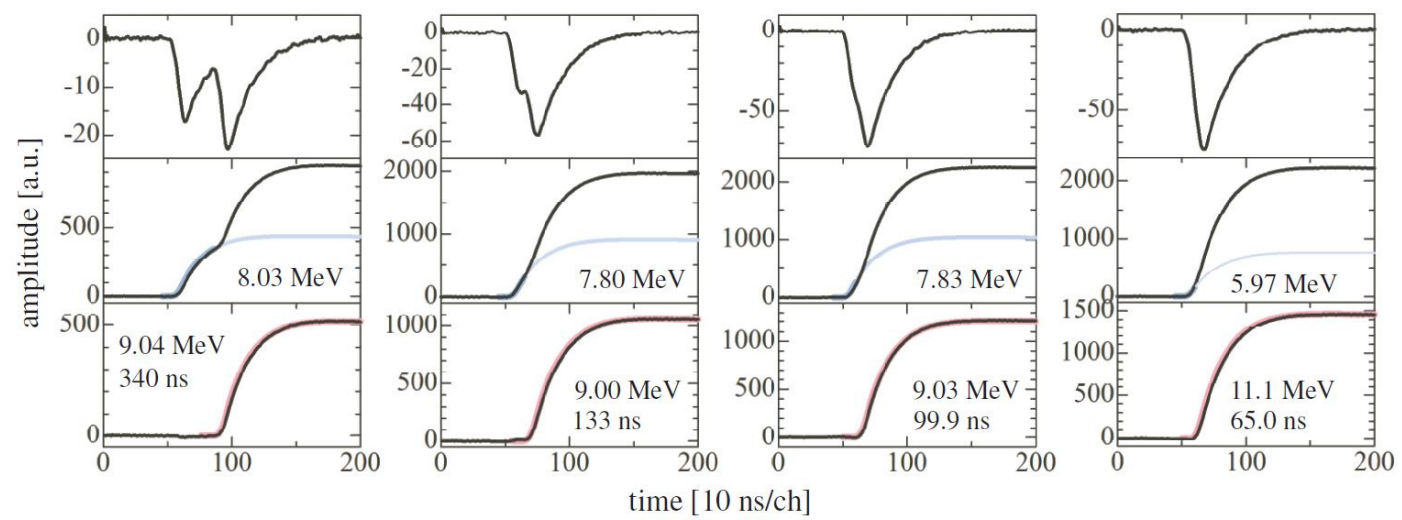

Figure 6 - Waveforms for the decay chain of ${ }^{220} \mathrm{Ac} \rightarrow{ }^{216} \mathrm{Fr}$.

The top graphs show the derivative waveforms, the middle graphs show the fittings of the first pulse $\left({ }^{220} \mathrm{Ac}\right)$, and the bottom graphs show the fittings of the second pulse $\left({ }^{216} \mathrm{Fr}\right)$

\section{Summary}

The PSA method enables the identification of pileup events and the individual deduction of the energy and $\Delta T$ of two sequential pulses. The efficiency of finding pileup events is almost $100 \%$ down to the $\Delta T$ of $300 \mathrm{~ns}$ in the $E_{1 s t} / E_{2 n d}$ range of $0.06-16$. The identification efficiency and energy as well as the $\Delta T$ accuracy depend on the $E_{1 s t} / E_{2 n d}$ and attain the best values at $\sim 1$. The derived energies for the short-lived $\alpha$ correlations in an online experiment show good agreement with the reference values. The present PSA aids in the accurate assignment of short-lived decay chains for future new-element searches.

\section{Acknowledgements}

The authors gratefully acknowledge everyone on the staffs of the RIBF and CNS for their contributions to the stable operation of the accelerator during this experiment. This work was supported by the RIKEN Junior Research Associate Program.

\section{References}

[1] K. Morita et al.. Experiment on the Synthesis of Element 113 in the ReactionBi( Zn.n) 113 // J. Phys. Soc. Jpn. - 2004. - Vol. 73. - P. 2593.
[2] K. Morita et al.. Observation of Second Decay Chain from113 // J. Phys. Soc. Jpn. - 2007. - Vol. 76. P.045001. 
[3] K. Morita et al.. New Result in the Production and Decay of an Isotope.113. of the 113th Element // J.Phys. Soc. Jpn. - 2012. - Vol. 81. - P. 103201.

[4] D. Kaji et al.. Gas-Filled Recoil Ion Separator GARIS-II // Nucl. Instr. Meth. B. - 2013. - Vol.. 317. P.311.

[5] Yu. Ts. Oganessian et al.. Attempt to Produce Element 120 in the Reaction // Phys. Rev. C. - 2009. Vol. 79. - P. 024603.

[6] A. Sobiczewski. Predictions for a Superheavy
Element 120 // Acta. Phys. Pol. B. - 2011. - Vol. 42. No 8. - P. 1871.

[7] S. Yamaki et al. Pulse Shape Analysis Using Flash-ADC for Short-Lived Decay of Superheavy Elements // JPS Conf. Proc. - 2015. - Vol. 6. - P. 030105.

[8] S. Yamaki et al. Pulse Shape Analysis for Short-Lived Decay of Superheavy Elements // RIKEN Accel. Prog. Rep. - 2014. - Vol. 48. - P. 215.

[9] R. K. Sheline et al. Shell Model Level Structure of ${ }^{216}$ Fr // Phys. Rev. C. - 1997. - Vol. 55. - P. 1162. 